\title{
Incoherent Production Reactions of Positive and Negative Ions in Matrix-Assisted Laser Desorption/Ionization
}

\author{
Bo-Hong Liu, ${ }^{\mathrm{a}, \mathrm{b}}$ Yuan Tseh Lee, ${ }^{\mathrm{a}, \mathrm{b}}$ and Yi-Sheng Wang ${ }^{\mathrm{b}}$ \\ a Department of Chemistry, National Taiwan University, Taipei, Taiwan R.O.C. \\ ${ }^{\mathrm{b}}$ Genomics Research Center, Academia Sinica, Taipei, Taiwan R.O.C.
}

Utilizing synchronized dual-polarity matrix-assisted laser desorption/ionization (MALDI) mass spectrometry, we found good evidence of the incoherent production of positive and negative matrix ions. Using thin, homogeneous 2,5-dehydroxybenzoic acid (DHB) matrix films, positive and negative matrix ions were found to appear at different threshold laser fluences. The presence of molecular matrix ions of single charge polarity suggests that the existence of DHB ion-pairs may not be a prerequisite in MALDI. Photoelectrons induced by the laser excitation may assist the production of negative DHB ions, as shown in experiments conducted with stainless steel and glass substrates. At high laser fluences, the relative yield of positive and negative matrix ions remained constant when homogeneous matrix films were used, but it fluctuated significantly with inhomogeneous crystal morphology. This result is also inconsistent with the hypothesis that matrix ion-pairs are essential primary ions. Evidence from both low and high laser fluences suggests that the productions of positive and negative matrix ions in MALDI may occur via independent pathways. (J Am Soc Mass Spectrom 2009, 20, 1078-1086) (c) 2009 American Society for Mass Spectrometry

$\mathrm{M}$ atrix-assisted laser desorption/ionization (MALDI) provides a convenient means to produce protein and peptide ions for mass analysis [1,2], but its application to the studies of biopolymers such as carbohydrates is still limited by insufficient ion yields $[3,4]$. It is generally accepted that the protonated matrix molecules are the essential initial ions responsible for the production of positively-charged analytes [5], normally via proton transfer reactions. But such a qualitative description is insufficient to provide guidelines for the optimization of experimental conditions for the wide variety seen in biological samples. The paucity of our knowledge of detailed ionization mechanisms makes the optimization of MALDI performance a highly empirical process and thus the primary bottleneck of this method. In the last decade, Karas and coworkers and Knochenmuss and coworkers have respectively established a cluster model $[6,7]$ and a two-step model [8-10] to explain MALDI. The two models are divergent in several aspects, including the origin of the initial ions that trigger the subsequent reactions, the role of ion-neutral reactions during material desorption, the contribution of photoelectrons in the reactions, etc. The two reaction models seem not converge over time mainly due to the insufficient information provided by conventional mass spectrometric methods. It is thus

Address reprint requests to Dr. Y.-S. Wang, Genomics Research Center, Academia Sinica, 128, Academia Road, Section 2, Nankang District, Taipei 115, Taiwan, R. O. C. E-mail: wer@gate.sinica.edu.tw desirable to develop our understanding of MALDI from novel perspectives, such as the correlation between primary positive and negative ions at the time of ionization.

A recent report based on a synchronized dual-polarity MALDI-TOF method stated that the spectral pattern of MALDI-generated positive ions were approximately equal to those of the negative ions for some moderate-size proteins [11]. There was thus some speculation over whether the positive and negative ions are produced in a pair-wise fashion in MALDI [12, 13], or whether they are the result of preformed ion-pairs in the crystal before laser irradiation [6]. More recently, Dashtiev and coworkers [14] systemically studied the correlation of positive and negative ions in MALDI using various proteins and matrices. Near-unity ratios between positive and negative total ion intensities were obtained, an understandable result if one assumes that the entire system remains electrically neutral. However, the authors surmised that thermodynamic modeling is inappropriate to predict quantitatively the particular ion abundances because of the insignificant change of cation-to-anion ratios with the change of molecular properties. This conclusion was challenged by Knochenmuss [15], who demonstrated that thermodynamic equilibria were complete in the systems discussed by Dashtiev and coworkers, and the unity relationship was due to the insignificant changes in the reaction Gibb's free energies. Nevertheless, in the thermodynamic modeling of Knochenmuss, the abun- 
dances of the initial positive and negative matrix ions were presumed to be equal. This presumption readily implies that the initial positive and negative matrix ions are produced pair-wise [16-18], or they are produced separately but fortunately yielded equal ionization efficiency. However, little investigation has addressed the correlations between the initial positive and negative ions, only the impact of free electrons on the positive ion yield has been considered experimentally [19-21]. Furthermore, charge neutralization may not occur in MALDI because while a significant amount of negative charges were considered to be photoelectrons [22, 23], their counterparts may not be free positive ions. Thus far, only some of the initial positive and negative matrix ions were studied individually in the literature $[6-8,10$, 24-27].

There is no conclusive evidence to determine whether the initial ions of opposite polarities are produced independently or via a closely correlated reaction pathway. Knochenmuss and coworkers proposed that energy pooling is a major reaction pathway responsible for the positive ion production $[9,28]$, and the source of negative charges may be the photoelectrons ejected from the matrix-metal hybrid molecular orbitals [22]. On the other hand, negative matrix ions may be produced by the attachment of low-energy electrons during material desorption processes, as reported previously by Pshenichnyuk and coworkers [29-31]. However, many papers proposed that the initial matrix ions may produced by the disproportionation of protons or electrons between adjacent matrix molecules, which involves the production of ion-pairs like $\mathrm{M}^{+}$and $\mathrm{M}^{-}$, or $[\mathrm{M}+\mathrm{H}]^{+}$ and $[\mathrm{M}-\mathrm{H}]^{-}[12,13,26,32]$, as shown below:

$$
\begin{array}{r}
2 \mathrm{M}+\mathrm{n} h \nu \rightarrow[\mathrm{MM}]^{*} \rightarrow \mathrm{M}^{+}+\mathrm{M}^{-} \\
\text {Electron disproportionation }
\end{array}
$$

$$
\begin{aligned}
& 2 \mathrm{M}+\mathrm{n} h v \rightarrow[\mathrm{MM}]^{*} \rightarrow[\mathrm{M}+\mathrm{H}]^{+}+[\mathrm{M}-\mathrm{H}]^{-} \\
& \text {Proton disproportionation }
\end{aligned}
$$

where $\mathrm{M}$ denotes matrix molecules and asterisk denotes excited states. Another apparent example is the cluster model, which suggests that the counter ions are embedded at various sites of small domains inside the crystal, and they are liberated from the solid phase after laser excitation [6, 33]. Notably, the lower abundance of negative matrix ions than positive matrix ions seems to be a general finding in some of these reports. Using a molecular dynamics (MD) simulation method, Knochenmuss and Zhigilei [34] suggested that the free electrons produced in MALDI escape from the dense plume region at the very early time, leaving a high density of positive ions dominant on the crystal surface. The authors concluded that those abundant positive ions on the surface result in the presence of more positive than negative ions in the spectra, but experimental supports of this hypothesis, especially based on any kind of synchronized dual polarity mass spectrometry, is still unavailable.

The main theme of the current work is to examine whether matrix ion pairs resulting from charge disproportionation reactions like eqs 1 and 2 exist in MALDI. A pure matrix system was selected to reduce complications due to charge-redistribution between multiple components. Synchronized dual-polarity MALDI-TOF mass spectrometry was utilized in this work to record all ions produced under various ionization conditions. The use of synchronized dual-polarity MALDI-TOF mass spectrometry has been successfully demonstrated previously [11] to allow unambiguous examination of counter ions; it allows all ions from every MALDI event to be recorded simultaneously. This feature was essential in this work because uncertainties due to low reproducibility were eliminated. The impacts of changes in laser fluence and crystal structure on the initial ion distribution of 2,5-dihydroxybenzoic acid (DHB) were examined because they are considered to be the major determinants of MS signal quality. DHB was selected as the target molecule because it is the best-studied matrix compound, both experimentally and theoretically [13, 35-38]. In the low fluence region, the appearance thresholds of positive and negative matrix ions should provide an unambiguous examination of the hypothesis of ion-pair production: the positive and negative ions should have identical thresholds if they are ion-pairs, whereas the presence of different thresholds would imply that ion-pairing is likely only a minor pathway. In high laser fluence region, the shotto-shot signal variations of the two polarities would be expected to be same if ion-pairing exists and is dominant in the reaction. In addition, the role of photoelectrons was also examined by changing the material of sample substrates from metal to glass, which significantly reduced the presence of photoelectrons.

\section{Experimental}

Two home-made synchronized dual-polarity mass spectrometers were used in this work, namely (1) a dual-polarity time-of-flight mass spectrometer (DTOFMS) reported previously [11], and (2) a simplified DTOFMS. Both instruments were equipped with MALDI ion sources at the instrument centers. In comparison with the previous version of the DTOFMS, three improvements are presented in this work: (1) increasing the ion kinetic energy from 5.9 to $15.2 \mathrm{keV}$, (2) incorporating delayed ion extraction (DE) to the ion source, and (3) improving the spectral quality by replacing the third harmonic photons of an Nd:YAG laser (355 $\mathrm{nm}$ ) by a tightly focused ( $\mathrm{f}=40 \mathrm{~mm}$ ) nitrogen laser beam (337 nm, 3 ns pulse width, VSL 337ND-S; Newport Co., Irvine, CA). The final size of the laser spot on the sample surface was roughly $90 \times 90 \mu \mathrm{m}$. Before ion extraction, both extraction electrodes adjacent to the sample electrode were biased at the same voltage as the sample electrode. DE was conducted by applying 
pulsed voltages of the same magnitude but opposite polarity simultaneously to the two extraction electrodes. The instrument shows mass resolving powers $(\mathrm{m} / \Delta \mathrm{m})$ of $\sim 475$ and $\sim 110$ for positive and negative modes at $m / z \sim 155$, respectively. Laser fluences of roughly $35-40 \mathrm{~mJ} / \mathrm{cm}^{2}$ were used in this instrument.

The simplified DTOFMS consisted of only a sample probe and two microchannel plate (MCP) detectors (Jordan TOF Products, Inc., Grass Valle, CA). The entire system was enclosed in a 6 in. cubic chamber. This simplified device minimized instrument biases that may cause unequal sensitivity of the two modes, especially at very low ion intensity near threshold laser fluence. The two MCPs were installed through two opposing vacuum ports, such that their entrance grids faced each other and were separated by roughly $6 \mathrm{~cm}$. The sample probe ( $3 \mathrm{~mm}$ o.d. and $15 \mathrm{~mm}$ long) was installed through another vacuum port and was located at the center of the space between the two MCPs. Laser beams excited the MALDI matrix crystals along the surface orthogonal of the sample substrates to eliminate any bias from the laser incidence angle. The sample surface was constantly kept at ground potential, whereas the two MCPs were biased with different voltages to extract positive and negative ions. For the negative ion $\mathrm{MCP}$, the biased voltages on the grid, entrance, middle, exit, and anode electrodes were respectively $+2200,+2200,+3200,+4200$, and $+4400 \mathrm{~V}$; for positive ion $\mathrm{MCP}$, the voltages were respectively $-2200,-2200,-1200,-200$, and $0 \mathrm{~V}$ with the same electrode sequence. Notably, a magnet was installed behind the source flange to deplete partially the photoelectrons to prevent the negative ion MCP from serious signal saturation problems. Baseline correction was conducted only for the negative spectra obtained using this instrument to eliminate the baseline drift due to the intense photoelectron signal. The magnetic field strength did not cause noticeable changes to the ion signals. During measurements, the pressure of the chamber was maintained at $7 \times 10^{-7}$ mbar by a turbomolecular pump. Data acquisition used a digital oscilloscope (Waverunner 6050A, 500 MHz; LeCory Inc., Chestnut Ridge, NY) and every spectrum was normally the accumulation of 50 laser shots. This instrument shared the same nitrogen laser as the DTOFMS but used a $250 \mathrm{~mm}$ focusing lens to focus the laser beam onto the sample surface. The final laser spot size was roughly $150 \times 150 \mu \mathrm{m}$ and the laser energies were measured outside the vacuum. The minimum fluence at which the matrix-associated features appeared after accumulation was reported as the threshold value in this work.

Two kinds of sample probes were used to study the contribution of photoelectrons. Stainless steel probes were used in most of the experiments to resemble the conventional MALDI conditions; the production of photoelectrons was significant in this case. To reduce the appearance of photoelectrons, a thin-layer of glass ( $\sim 0.5 \mathrm{~mm}$ thick) covered the stainless steel surface was

used as the sample substrate for the same experiments. All probes were cleaned thoroughly in sonication bath sequentially with detergent, water, spectral grade acetone, and distillated deionized water. They were dried on a $70{ }^{\circ} \mathrm{C}$ heating plate before use. During measurements, the sample probes were constantly rotated with slow speed so that every laser shot examined a fresh sample position.

2,5-DHB was purchased from Fluka (Buchs, Switzerland) and was used without further purification. It was dissolved in acetonitrile/water solution (50:50 vol:vol) to a concentration of roughly $0.1 \mathrm{M}$. The sample was deposited onto the probe in two ways: (1) as a homogeneous matrix film on the sample surface with no apparent crystals; and (2) with obvious matrix crystals on the sample surface. To prepare the homogeneous matrix film, $0.1 \mu \mathrm{L}$ of sample solution was deposited at the center of the probe for air drying on the $70^{\circ} \mathrm{C}$ heating plate. The amount of sample deposited on the probe in this condition was $\sim 1.5 \mu \mathrm{g}$, as estimated by UV absorption spectroscopy to the solution. After drying, $0.5 \mu \mathrm{L}$ of acetone was deposited on the probe allowing the matrix crystal to redissolve and dry rapidly to form a homogeneous matrix film. To prepare the inhomogeneous matrix crystals, $0.2 \mu \mathrm{L}$ of sample was deposited onto the sample surface. The sample liquid was then air-dried on the $70{ }^{\circ} \mathrm{C}$ heating plate for about $1 \mathrm{~min}$ to form inhomogeneous crystal structures at the surface center.

\section{Results and Discussion}

Although it has been claimed that the MALDI mass spectra may not reveal useful primary ionization mechanisms [24, 32], new insight into these mechanisms may still be extracted from the correlation of spectral features between two opposite polarities. The present work focuses on a simple condition where no guest (analyte) molecules are involved, thus the charged species should associate only with the matrix and should be eventually detected. To obtain accurate information, the experiments under high and low laser fluence were conducted respectively by using the DTOFMS and the simplified DTOFMS. The impact of the change of sample homogeneity to the yield of positive and negative ions is

(a)

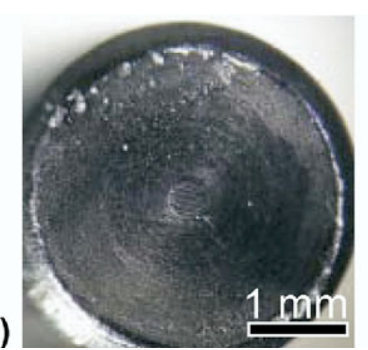

Figure 1. Homogeneous matrix film (a) and inhomogeneous matrix crystal (b) on sample probes. 
also tested: the homogeneous matrix film covered the entire sample probe surface without noticeable crystals, whereas the inhomogeneous matrix showed apparent needle-like crystals, as shown in Figure 1. The needles showed widths of roughly 20-50 $\mu \mathrm{m}$ and lengths of about $100-700 \mu \mathrm{m}$.

In the cases of simplified DTOFMS, the data allows us to determine the production of ions at very low laser fluences. With minimized dimensions, the simplified DTOFMS reduces both the time and distance that the ions experience after ionization, which consequently reduces the ion loss. This unique feature drastically minimizes the uncertainty when detecting very low ion fluxes using regular TOF instruments. At the threshold fluences, the matrix-associated features obtained by using the simplified DTOFMS were barely detected with less than four shots, and their signal-to-noise ratio became higher than seven after accumulating 50 laser shots. Therefore, the threshold phenomena reported here should be very close to the true reaction results. When high ion flux is produced by using high laser fluences, accumulation of mass spectra is no longer advantageous due to signal saturation problem.

It is important to note that the sensitivity of the MCP for positive and negative ions is comparable with the acceleration energy used in this work, as reported previously by Stephen and Peko [39, 40]. Exchanging the polarity of the two MCP detectors produced the same experimental results, indicating the two detectors possessed roughly the same detection efficiency. With the simplified DTOFMS, we managed to verify the spectral features with significant mass differences such as photoelectrons, surface metals, matrix-associated species, and secondary ions. We did not attempt precise peak assignments because of insufficient mass resolving power.

The DTOFMS was used for the studies under high laser fluences with both intense positive and negative ions present in the plume; the ion-pairs were examined by analyzing the correlations of signal variations between the positive and the negative modes, especially when various sample morphologies were used. Additionally, it is important to identify every spectral feature when strong and complex ion signals present in the spectra. Comparisons of positive and negative ion spectra are only meaningful when the two modes are recorded in a simultaneous fashion as described in this work; conventional polarity-switching methods were unable to deliver unambiguous signal correlations due to low shot-to-shot reproducibility problems of MALDI.

Typical DHB mass spectra obtained by the DTOFMS exhibited several features of DHB ions in both polarities and fragments only in the positive mode (Figure 2). The intensities of matrix ions were determined by integrating the spectral areas of matrix-associated features, including molecular ions, cationic matrix ions, and their major fragments. The matrix-associated negative ions (m-negative ions) observed in this work include the signals between $\mathrm{m} / \mathrm{z}$ 152-154, in which the $\left[\mathrm{M}-\mathrm{H}_{2}\right]^{\cdot-}$

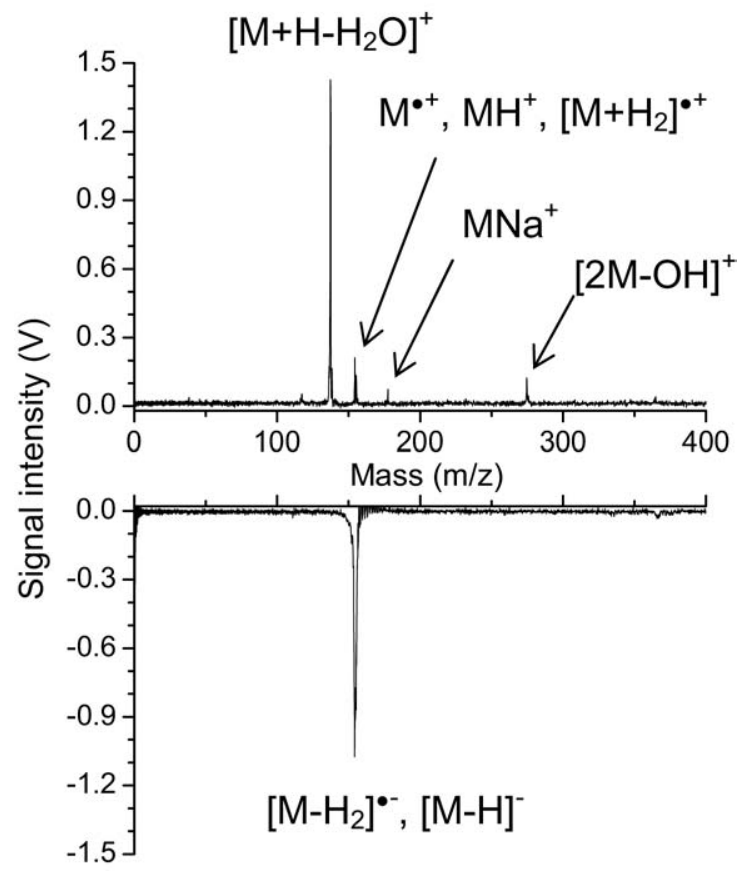

Figure 2. Synchronized positive and negative ion spectra of 2,5-DHB obtained by DTOFMS. The features in positive mode include abundant dehydroxy fragments and low abundance cationic and cluster ions. The negative spectrum contained only features at the molecular ion region.

and $[\mathrm{M}-\mathrm{H}]^{-}$are the most abundant features. On the other hand, the matrix-associated positive ions (mpositive ions) include the features at $\mathrm{m} / \mathrm{z} 137([\mathrm{M}+\mathrm{H}-$ $\left.\left.\mathrm{H}_{2} \mathrm{O}\right]^{+}\right), 154-156\left(\mathrm{M}^{\bullet+},[\mathrm{M}+\mathrm{H}]^{+},\left[\mathrm{M}+\mathrm{H}_{2}\right]^{\bullet+}\right)$, and 177 $\left([\mathrm{M}+\mathrm{Na}]^{+}\right)$. Commonly observed impurities such as alkali metal ions in the low molecular weight region were excluded in the analyses except as mentioned otherwise.

\section{Appearance Thresholds of Ions (Low Laser Fluence)}

When using stainless steel probes and homogeneous matrix films, the m-negative ions had an appearance threshold at a laser fluence of roughly $11.1 \mathrm{~mJ} / \mathrm{cm}^{2}$, which was lower than that of $\mathrm{m}$-positive ions at roughly $13.3 \mathrm{~mJ} / \mathrm{cm}^{2}$. Remarkably, the absence of m-positive ions readily indicates that the matrix ion-pairs are not a prerequisite for the production of initial matrix ions in MALDI. Figure 3a shows the positive and negative ion spectra with a laser fluence of $\sim 12 \mathrm{~mJ} / \mathrm{cm}^{2}$, at such fluence the m-positive ions are still undetectable. Note that the spectral features appearing in the positive spectra (top panel of Figure 3a) are presumably metal ions that came from (1) direct MALDI reactions and (2) secondary metal ions ejected from the negative ion MCP surface. Such secondary metal ions are probably ejected when photoelectrons of the MALDI reaction impinge on the MCP surface. Those secondary metal ions were accelerated toward the positive ion MCP detector and produced signals, as verified by TOF predictions 


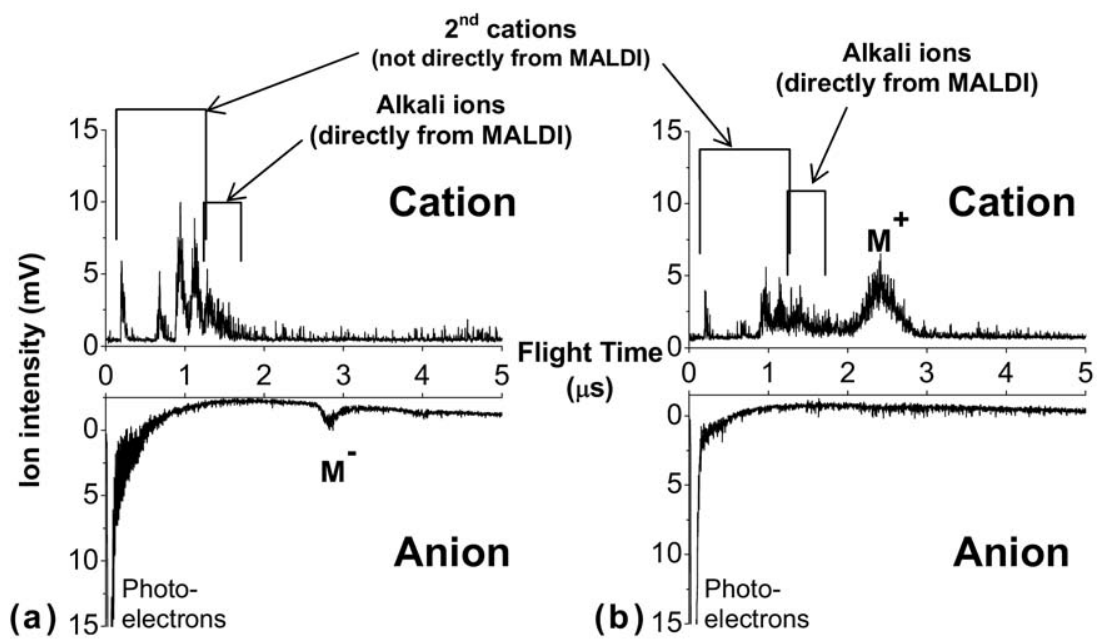

Figure 3. Positive and negative ion spectra obtained by simplified DTOFMS. The negative matrix ions had a lower appearance threshold than positive matrix ions on stainless steel substrates (a); the positive matrix ions had a lower threshold than negative matrix ions if glass substrates were used (b). The over-scale features in the negative spectra are photoelectrons, and the features in the low TOF region of positive modes correspond to secondary cations emitted from the negative ion MCP due to bombardment by the photoelectrons.

based on ion trajectory simulations. A good support of this assignment is that there was no secondary metal ion signal when the biased voltage of the negative ion MCP was reduced; under that condition the energy of the photoelectrons is likely insufficient to induce the ejection of secondary ions from the surface of MCP. Conducting the experiment without matrix on the substrate surface produced the same secondary metal ions.

The spectral features in Figure 3a show that the overall charge of the system was not neutral at the threshold laser fluences. If neglecting the secondary metal ions, the integrated negative charges significantly outweighed the positive charges. The difference was very pronounced due to the intense photoelectrons. It should be noted that the exact intensities of the photoelectrons were actually higher than that displayed in the figure due to the interference of the magnetic field. The early presence of m-negative ions suggests that the MD simulation by Knochenmuss and coworkers predicting a dominance of positive ions at the crystal surface and in the MALDI spectra [34] is probably not a general fact. A possible reason for low positive charges is that the counterparts of the photoelectrons may be trapped in the solid phase and eventually diminish in the source region. Such counter parts may be the metal substrates, bottom-layer matrices, or big chunks of matrix clusters that are not visible in MALDI spectra, as proposed elsewhere in the literature [14, 41]. Therefore, the production of m-negative ions may proceed via electron-attachment reactions $[29,31]$ because the ejection of both neutral molecules and photoelectrons occurred at fluences lower than the ion appearance threshold [42].

The role of photoelectrons was further examined by using homogeneous matrix films on the glass substrate, which produced roughly half as many photoelectrons as the stainless steel substrates. The source of the photoelectron from this dielectric surface may be the matrix molecules or the ambient gas molecules adsorbed on the surface. Ambient gas adsorption is a common phenomenon especially when the surface is exposed to ambient or high vacuum condition. Removal of the adsorbates requires sophisticated treatments to the surface and an ultra-high vacuum system; such procedures were not conducted in this work because they deviate from the conventional MALDI conditions. The result presented here provides a qualitative inspection into the change of spectral features with that of photoelectron yield; the quantitative interpretation of MALDI is still difficult due to great complexity.

With the glass substrate, the resultant ion appearance thresholds were $13.3 \mathrm{~mJ} / \mathrm{cm}^{2}$ for m-positive ions and $15.5 \mathrm{~mJ} / \mathrm{cm}^{2}$ for $\mathrm{m}$-negative ions. Interestingly, the critical fluence for $\mathrm{m}$-positive ions remained the same as with stainless steel substrates, but for m-negative ions with the glass substrate, the threshold increased considerably and was now higher than for m-positive ions. Between 13.3 and $15.5 \mathrm{~mJ} / \mathrm{cm}^{2}$, only m-positive but no $\mathrm{m}$-negative ions were present in the spectra (Figure $3 b$ ). However, the integrated ion intensities with glass substrate showed larger variations than those from stainless steel substrates. The large intensity variations may due to the interference of dielectric substrates, such as the periodic accumulation and quenching of charges on the substrate surface.

Above the critical laser fluences, the matrix ion signals of both polarities increased rapidly but showed somewhat different rates on stainless steel and glass 

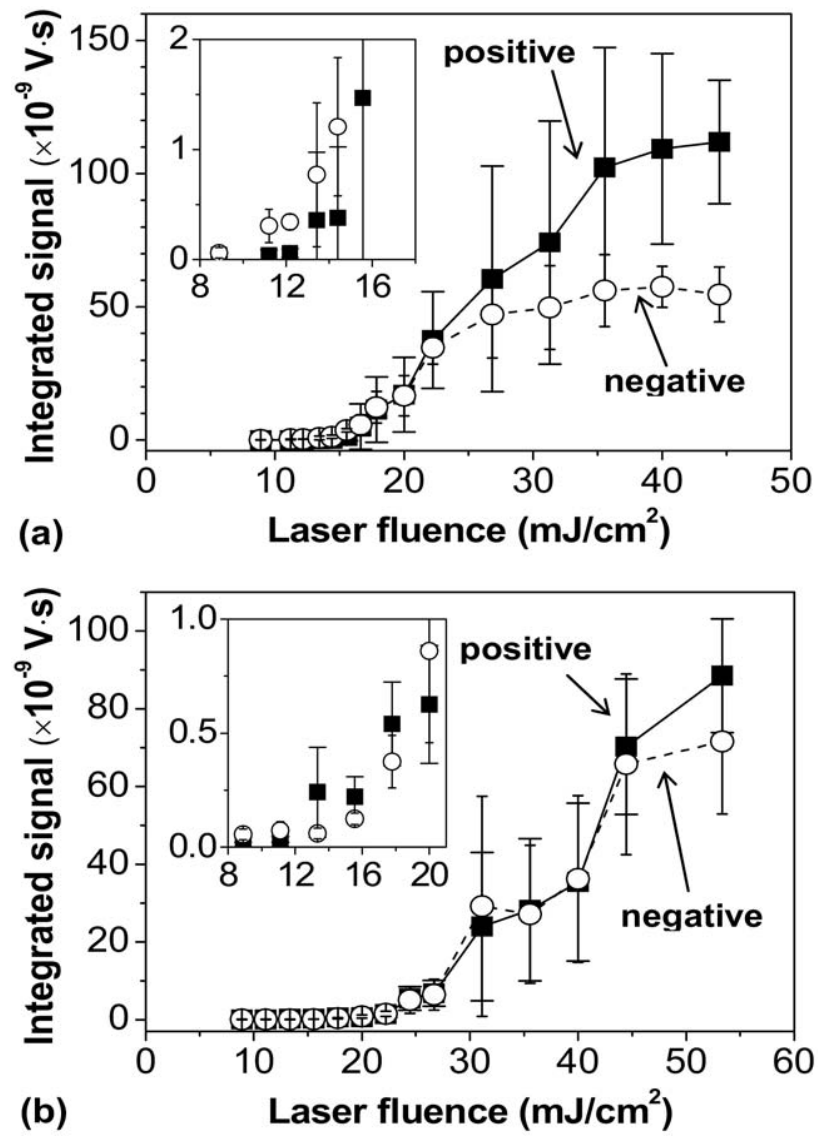

Figure 4. Integrated signal intensities versus laser energy. (a) Signals of molecular ion regions with stainless steel substrates; (b) signals of molecular ion regions with glass substrates; [filled square $]=$ positive ions; [open circle] $=$ negative ions. The insets illustrate the data only near the ion appearance thresholds.

substrates (Figure $4 a, b$ ). The m-positive ion intensities with stainless steel substrates increased faster than the $\mathrm{m}$-negative ion intensities (Figure 4a). Because the mnegative ions appeared first at lower laser fluences than the m-positive ions, an intensity crossing point occurred at roughly $20.0 \mathrm{~mJ} / \mathrm{cm}^{2}$. When above $35.6 \mathrm{~mJ} / \mathrm{cm}^{2}$ the $\mathrm{m}$-negative ion signal reached a plateau, whereas the m-positive ion intensity still increased although at a lower rates than in the low laser fluence region. The difference of signal intensity was pronounced in high laser fluence region; the m-negative ion intensity was about half that of m-positive ions at a laser fluence of $44.4 \mathrm{~mJ} / \mathrm{cm}^{2}$.

Such differences in ion abundance between the two polarities vanished when the glass substrate was used (Figure $4 \mathrm{~b}$ ). With glass substrate, the intensity curves of the two polarities showed two distinct differences from the curves obtained using stainless steel substrates. First, the m-positive and m-negative ion curves overlaid with each other with good consistency in ion intensity, and they both progressed with a lower slope than with stainless steel substrate. Second, the intensities increased steadily even at $53.3 \mathrm{~mJ} / \mathrm{cm}^{2}$, whereas those from the stainless steel substrate had plateaued at that fluence.

Under higher laser fluences, the absolute intensity of the m-negative ions with glass substrate was higher than with stainless steel substrates, but the m-positive ions showed the opposite trend. It is thus considered that the contribution of the photoelectrons from a stainless steel surface may play an important role in the determination of the charge distribution of desorbed DHB. The low m-negative ion yield with the stainless steel substrate under high laser fluences might result from an increase in the amount of photoelectrons that develop serious space charge problems, and that may also shift the energy of photoelectrons to above the appropriate electronattachment energy regions. Such changes in space charge and electron energy would reduce the production probability of m-negative ions. Furthermore, the contribution of multiphoton ionization processes of matrix molecules may dominate under high laser fluences and produce more m-positive ions.

The trends of the intensity curves show similar qualities even when all charged species in the entire spectral range are taken into account, as depicted in Figure 5. One of the major changes from the data of matrix-only analyses discussed above is that the distinction between the curves of total positive ( $t$-positive) and total negative ( $t$-negative) ions (including the photoelectrons) became more pronounced. Qualitatively, the $t$ negative ion integration started from a non-zero value at the minimum fluence value due to the presence of photoelectrons, whereas the $t$-positive ions show zero intensity at the same range. As the laser fluence increase, the $t$-positive curves increased faster than those of the $t$-negative ions. The crossing point with stainless steel substrate was roughly $17.8 \mathrm{~mJ} / \mathrm{cm}^{2}$ and with glass

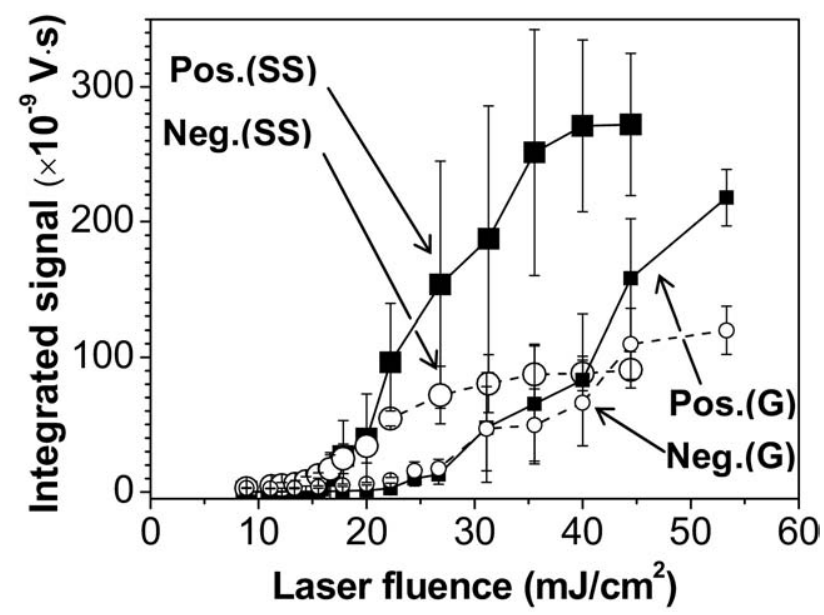

Figure 5. Integrated total charge intensities versus laser energy with stainless steel (SS) and glass (G) substrates; [large filled square $]=$ positive charges from stainless steel substrate; [large open circle] $=$ negative charges from stainless steel substrate; [small filled square] = positive charges from glass substrate; [small open circle] $=$ negative charges from glass substrate. 
substrate was $31.1 \mathrm{~mJ} / \mathrm{cm}^{2}$. Because of the significant differences in the values of the total charge integration when using stainless steel substrates, charge neutralization was not considered to be reached in the MALDI reaction of this condition. This result seems to contradict with the result reported by Dashtiev and coworkers [14], but possibly the laser fluence used by those authors was not high enough to observe the difference in total ion yield. In fact, the similar ion yields between positive and negative ions from the glass substrate agree with the observation in the same literature.

With inhomogeneous crystals, there were no confident conclusions to be made for both the stainless steel and the glass substrates because the observed appearance thresholds fluctuated severely in these measurements. We could not predict whether positive or negative matrix ions may appear first, and in some cases ions of both polarities appeared at the same fluence. Although this observation may imply that the changes in crystal structure can alter the production reaction of positive and negative ions, the bad reproducibility prevents it from a conclusive interpretation. Further experimental information regarding the impact of crystal structure on the production of positive and negative ions is discussed in the next section.

\section{Signal Variations Between the Two Charge Polarities (High Laser Fluence)}

Use of the DTOFMS allowed the identities of all spectral features to be determined, and all of them are included in the statistical analyses except photoelectrons. The typical mass spectra are displayed in Figure 2 and the integrated signal intensities are shown in Figure 6. It is noteworthy that the mass spectra obtained in this series of experiments represent the results of single laser shots, and the data included in the statistical analyses were collected from every spectrum.

For the homogeneous thin matrix film with the stainless steel substrate, the obtained mass spectra show good reproducibility on every crystal position. Under this source condition, the anion-to-cation intensity ratios show a median of 2.77 and a relative standard deviation (RSD) of $22 \%$, as displayed in Figure 6a. Note that the anion-to-cation ratio would change if different laser fluence is used for the measurements. Small RSD values within $10 \%-23 \%$ were also found when analyzing the absolute ion intensity. Different sample probes with similar thin sample film result in similar average values and also small standard deviations. The implications of the result are thus manifold: first, the reproducibility of the DTOFMS when operating at the same instrument settings is better than $76 \%$. Note that the variation reported here also involved the sample homogeneity issue. Second, the morphologies of the thin sample film used in this work were uniform microscopically because the relative abundance of the positive

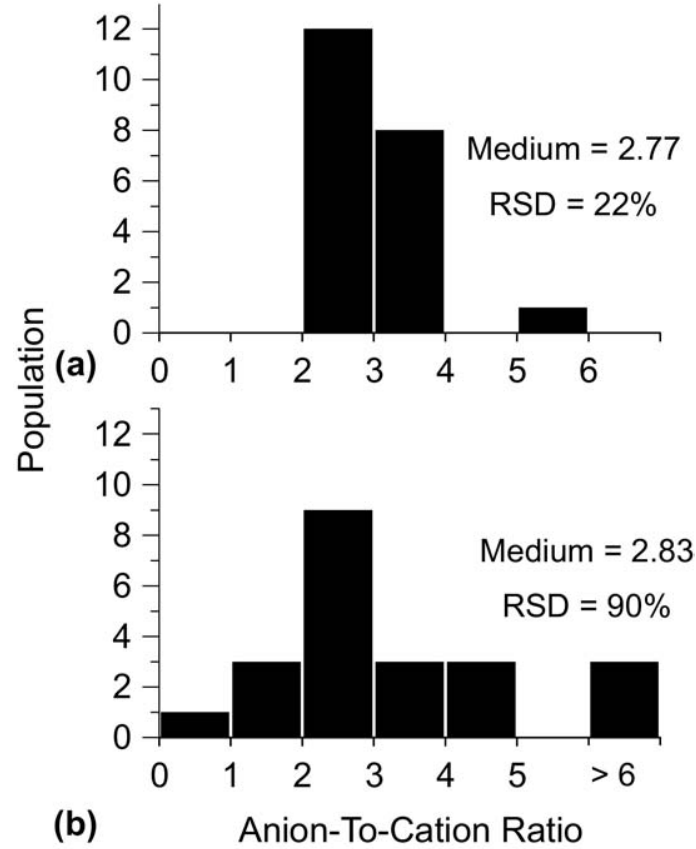

Figure 6. Histograms of anion-to-cation matrix ion intensity ratios of 21 iterations with (a) homogeneous and (b) inhomogeneous matrix samples. All data within the same integer segment are presented by a single bar. The homogeneous film shows a sharply defined population with small standard deviation, while the inhomogeneous crystal shows wide population range with large variations.

and negative ions exhibits good consistency and reproducibility in most of the sample positions.

For inhomogeneous crystallized matrix, however, the obtained mass spectra showed scattered absolute ion intensities (RSD $=53 \%-75 \%$ ) and anion-to-cation ratios, as displayed in Figure $6 \mathrm{~b}$. Under the same condition as that in Figure 6a, the median with inhomogeneous matrix crystals was 2.83 and the RSD was $90 \%$, a similar median as in Figure 6a but a much higher standard deviation. This indicates that changes of crystal morphology have a strong impact on the resultant anion-to-cation ratios. Because the crystal structure of the inhomogeneous matrix crystal changes substantially in different location, the initial ionization conditions may also change accordingly. For example, the effective laser fluence received by the crystal may change with the surface profile of the crystal. The surface geometry of the crystal may also change the desorption behaviors and consequently change the ionneutral collision conditions.

The observed results imply that the correlation of production reactions for positive and negative ions is weak. That interpretation means that the positive and negative ions may be produced by distinct reaction pathways with little connection; otherwise the variation of positive and negative signal intensities should converge to some degree. The crystal morphology is thus one of the important parameter that changes the ion yield. 


\section{Conclusions}

For 2,5-DHB, the observed different ion appearance thresholds and signal variations suggest that the production reactions for initial positive and negative matrix ions may be incoherent. At or near the ion appearance thresholds, the negative matrix ions had a lower appearance laser fluence than the positive matrix ions when stainless steel substrates and homogeneous matrix films were used. In contrast, the positive matrix ions had a lower appearance fluence with glass substrates. The absence of matrix ions of certain polarity under low laser fluences indicates that matrix ion-pair production may not be a requisite in MALDI reactions. The large amount of photoelectrons emitted from stainless steel surfaces may be responsible for such threshold differences, in which the matrix negative ions may be largely produced via electron-capture ionization shortly after the laser pulses. Under high laser fluences, although positive and negative matrix ions were present simultaneously, their shot-to-shot intensity variations showed no apparent correlation. This result suggests again that the ion-pair may not be an essential part of the reaction, and the abundant positive matrix ions may produced via an independent pathway such as energy pooling process. All these findings do not support the hypothesis that the existence of matrix ion pairs result from the disproportionation of protons or electrons after laser excitation, unless they may exist briefly but quench immediately and do not contribute to the final ionic products. On the other hand, the previous prediction of more positive ions than negative ions on crystal surface also deviates from the observation of this work. We propose that the previous observations of similar ion distributions in positive and negative ion modes may be mainly due to the averaging out of shot-to-shot signal variation, and perhaps also to the selection of laser fluence ranges that give comparable abundances. As a consequence, chemical equilibrium between positive and negative ions arising from equal abundance of positive and negative matrix ions is probably a premature assumption and needs further examinations. The impacts of laser fluence, sample morphology, matrixto-analyte ratios, and substrate materials on the yield of positive and negative ions may all need to be reconsidered for precise numerical interpretations. To eliminate the polarity bias of conventional methods, conducting experiments using synchronized dual-polarity ion detection approaches is highly recommended.

\section{Acknowledgments}

The authors acknowledge financial support from the Genomics Research Center, Academia Sinica, and the National Science Council of the Republic of China, Taiwan (contract number NSC 96-2113-M-001-027-MY2). The authors thank Professor ShengHsian Lin of the Institute of Atomic and Molecular Sciences and Professor Yi-Hau Chen of the Institute of Statistics of Academia Sinica for insightful discussions. The corresponding author thanks Professor Franz Hillenkamp for helpful suggestions.

\section{References}

1. Karas, M.; Hillenkamp, F. Laser desorption ionization of proteins with molecular masses exceeding 10,000 daltons. Anal. Chem. 1988, 60(20), 2299-2301.

2. Hillenkamp, F.; Peter-Katalinic, J. MALDI MS: A practical guide to instrumentation, methods and applications; Wiley-VCH: Weinheim, 2007.

3. Harvey, D. Analysis of carbohydrates and glycoconjugates by matrixassisted laser desorption/ionization mass spectrometry: An update covering the period 2001-2002. Mass Spectrom. Rev. 2008, 27(2), 125-201.

4. Harvey, D. Matrix-assisted laser desorption/ionization mass spectrometry of carbohydrates. Mass Spectrom. Rev. 1999, 18(6), 349-450.

5. Knochenmuss, R.; Zenobi, R. MALDI ionization: The role of in-plume processes. Chem. Rev. 2003, 103(2), 441-452.

6. Karas, M.; Gluckmann, M.; Schafer, J. Ionization in matrix-assisted laser desorption/ionization: singly charged molecular ions are the lucky survivors. J. Mass Spectrom. 2000, 35(1), 1-12.

7. Karas, M.; Kruger, R. Ion formation in MALDI: The cluster ionization mechanism. Chem. Rev. 2003, 103(2), 427-439.

8. Knochenmuss, R. A quantitative model of ultraviolet matrix-assisted laser desorption/ionization. J. Mass Spectrom. 2002, 37(8), 867-877.

9. Knochenmuss, R. A quantitative model of ultraviolet matrix-assisted laser desorption/ionization including analyte ion generation. Anal. Chem. 2003, 75(10), 2199-2207.

10. Knochenmuss, R. Ion formation mechanisms in UV-MALDI. Analyst. 2006, 131(9), 966-986.

11. Tsai, S.-T.; Chen, C. W.; Huang, L. C. L.; Huang, M. C.; Chen, C. H.; Wang, Y.-S. Simultaneous mass analysis of positive and negative ions using a dual-polarity time-of-flight mass spectrometer. Anal. Chem. 2006, 78(22), 7729-7734.

12. Zenobi, R.; Knochenmuss, R. Ion formation in MALDI mass spectrometry. Mass Spectrom. Rev. 1998, 17(5), 337-366.

13. Bourcier, S.; Bouchonnet, S.; Hoppilliard, Y. Ionization of 2,5dihydroxybenzoic acid (DHB) matrix-assisted laser desorption ionization experiments and theoretical study. Int. J. Mass Spectrom. 2001, 210/211(1/3), 59-69.

14. Dashtiev, M.; Wafler, E.; Rohling, U.; Gorshkov, M.; Hillenkamp, F. Zenobi, R. Positive and negative analyte ion yield in matrix-assisted laser desorption/ionization. Int. J. Mass Spectrom. 2007, 268(2/3), 122 130.

15. Knochenmuss, R. Positive/negative ion ratios and in-plume reaction equilibria in MALDI. Int. J. Mass Spectrom. 2008, 273(1/2), 84-86.

16. Breuker, K.; Knochenmuss, R.; Zhang, J.; Stortelder, A.; Zenobi, R. Thermodynamic control of final ion distributions in MALDI: in-plume proton transfer reactions. Int. J. Mass Spectrom. 2003, 226(1), 211-222.

17. Knochenmuss, R.; Karbach, V.; Wiesli, U.; Breuker, K.; Zenobi, R. The matrix suppression effect in matrix-assisted laser desorption/ionization: Application to negative ions and further characteristics. Rapid Commun. Mass Spectrom. 1998, 12(9), 529-534.

18. Breuker, K.; Knochenmuss, R.; Zenobi, R. Gas-phase basicities of deprotonated matrix-assisted laser desorption/ionization matrix molecules. Int. J. Mass Spectrom. 1999, 184(1), 25-38.

19. Frankevich, V.; Zhang, J.; Dashtiev, M.; Zenobi, R. Production and fragmentation of multiply charged ions in 'electron-free' matrix-assisted laser desorption/ionization. Rapid Commun. Mass Spectrom. 2003, 17(20), 2343-2348.

20. Frankevich, V. E.; Zhang, J.; Friess, S. D.; Dashtiev, M.; Zenobi, R. Role of electrons in laser desorption/ionization mass spectrometry. Anal. Chem. 2003, 75(22), 6063-6067.

21. Dashtiev, M.; Frankevich, V.; Zenobi, R. Kinetic energy of free electrons affects MALDI positive ion yield via capture cross-section. J. Phys. Chem. A. 2006, 110(3), 926-930.

22. Knochenmuss, R. Photoionization pathways and free electrons in UVMALDI. Anal. Chem. 2004, 76(11), 3179-3184.

23. Cui, W.; Hu, Y.; Lifshitz, C. Time resolved photo dissociation of small peptide ions-combining laser desorption with ion trap/reflectron TOF mass spectrometry. Eur. Phys. J. D. 2002, 20(3), 565-571.

24. Papantonakis, M. R.; Kim, J.; Hess, W. P.; Haglund, R. F. What do matrix-assisted laser desorption/ionization mass spectra reveal about ionization mechanisms? J. Mass Spectrom. 2002, 37(6), 639-647.

25. Land, C. M.; Kinsel, G. R. The mechanism of matrix to analyte proton transfer in clusters of 2,5-dihydroxybenzoic acid and the tripeptide VPL. J. Am. Soc. Mass Spectrom. 2001, 12(6), 726-731.

26. GimonKinsel, M.; Preston-Schaffter, L. M.; Kinsel, G. R.; Russell, D. H. Effects of matrix structure/acidity on ion formation in matrix-assisted laser desorption ionization mass spectrometry. J. Am. Chem. Soc. 1997, 119(10), 2534-2540.

27. Lin, Q.; Knochenmuss, R. Two-photon ionization thresholds of matrixassisted laser desorption/ionization matrix clusters. Rapid Commun. Mass Spectrom. 2001, 15(16), 1422-1426.

28. Karbach, V.; Knochenmuss, R. Do single matrix molecules generate primary ions in ultraviolet matrix-assisted laser desorption/ionization. Rapid Commun. Mass Spectrom. 1998, 12(14), 968-974.

29. Asfandiarov, N. L.; Pshenichnyuk, S. A.; Fokin, A. I.; Lukin, V. G. Fal'ko, V. S. Electron capture negative ion mass spectra of some typical matrix-assisted laser desorption/ionization matrices. Rapid Commun. Mass Spectrom. 2002, 16(18), 1760-1765.

30. Pshenichnyuk, S. A.; Asfandiarov, N. L.; Fal'ko, V. S.; Lukin, V. G. Temperature dependence of dissociative electron attachment to mole- 
cules of gentisic acid, hydroquinone and p-benzoquinone. Int. J. Mass Spectrom. 2003, 227(2), 281-288.

31. Pshenichnyuk, S. A.; Asfandiarov, N. L.; Fal'ko, V. S.; Lukin, V. G. Temperature dependencies of negative ions formation by capture of low-energy electrons for some typical MALDI matrices. Int. J. Mass Spectrom. 2003, 227(2), 259-272.

32. Knochenmuss, R.; Stortelder, A.; Breuker, K.; Zenobi, R. Secondary ion-molecule reactions in matrix-assisted laser desorption/ionization. J. Mass Spectrom. 2000, 35(11), 1237-1245.

33. Kruger, R.; Karas, M. Formation and fate of ion pairs during MALDI analysis: Anion adduct generation as an indicative tool to determine ionization processes. J. Am. Soc. Mass Spectrom. 2002, 13(10), 1218-1226.

34. Knochenmuss, R.; Zhigilei, L. V. Molecular dynamics model of ultraviolet matrix-assisted laser desorption/ionization including ionization processes. J. Phys. Chem. B. 2005, 109(48), 22947-22957.

35. Strupat, K.; Kar, M.; Hillenkamp, F. 2,5-Dihydroxybenzoic acid-a new matrix for laser desorption ionization mass-spectrometry. Int. J. Mass Spectrom. Ion Processes. 1991, 111, 89-102.

36. Horneffer, V.; Dreisewerd, K.; Ludemann, H. C.; Hillenkamp, F.; Lage, M.; Strupat, K. Is the incorporation of analytes into matrix crystals a prerequisite for matrix-assisted laser desorption/ionization mass spec- trometry? A study of five positional isomers of dihydroxybenzoic acid Int. J. Mass Spectrom. 1999, 187, 859-870.

37. Wallace, W. E.; Arnould, M. A.; Knochenmuss, R. 2,5-Dihydroxybenzoic acid: Laser desorption/ionization as a function of elevated temperature. Int. J. Mass Spectrom. 2005, 242(1), 13-22.

38. Mormann, M.; Bashir, S.; Derrick, P. J.; Kuck, D. Gas-phase basicities of the isomeric dihydroxybenzoic acids and gas-phase acidities of their radical cations. J. Am. Soc. Mass Spectrom. 2000, 11(6), 544-552.

39. Stephen, T. M.; Peko, B. L. Absolute calibration of a multichannel plate detector for low energy O, O-, and O+. Rev. Sci. Instrum. 2000, 71(3), 1355-1359.

40. Peko, B. L.; Stephen, T. M. Absolute detection efficiencies of low energy $\mathrm{H}, \mathrm{H}-, \mathrm{H}+, \mathrm{H}-2(+)$, and $\mathrm{H}-3(+)$ incident on a multichannel plate detector. Nucl. Instrum. Methods Phys. Res. Sect. B Beam Interact. Mater. Atoms. 2000, 171(4), 597-604.

41. Krutchinsky, A.; Chait, B. On the nature of the chemical noise in MALDI mass spectra. J. Am. Soc. Mass Spectrom. 2002, 13(2), 129-134.

42. Spengler, B.; Bahr, U.; Karas, M.; Hillenkamp, F. Post-ionization of laser-desorbed organic and inorganic-compounds in a time of flight mass-spectrometer. Anal. Instrum. 1988, 17(1/2), 173-193. 